\title{
Effect of Nordic Hamstring Exercise Training on Knee Flexors Eccentric Strength and Fascicle Length: A Systematic Review and Meta-Analysis
}

\author{
Diulian Muniz Medeiros, César Marchiori, and Bruno Manfredini Baroni
}

\begin{abstract}
Context: Nordic hamstring exercise (NHE) has been widely employed to prevent hamstring strain injuries. However, it is still not clear which adaptations are responsible for the NHE preventive effects. Objectives: The aim of this study was to investigate the effects of NHE on knee flexors eccentric strength and fascicle length. Evidence Acquisition: The search strategy included MEDLINE, PEDro, and Cochrane CENTRAL from inception to April 2020. Randomized clinical trials that have analyzed the effects of NHE training on hamstring eccentric strength and/or fascicle length were included. Evidence Synthesis: From the 1932 studies identified, 12 were included in the systematic review, and 9 studies presented suitable data for the meta-analysis. All studies demonstrated strength increments in response to NHE training (10\%-15\% and $16 \%-26 \%$ in tests performed on the isokinetic dynamometer and on the NHE device, respectively), as well as significant enhancement of biceps femoris long head fascicle length (12\%-22\%). Meta-analysis showed NHE training was effective to increase knee flexors eccentric strength assessed with both isokinetic tests $(0.68 ; 95 \%$ confidence interval, 0.29 to 1.06$)$ and NHE tests $(1.11 ; 95 \%$ confidence interval, 0.62 to 1.61). NHE training was also effective to increase fascicle length $(0.97 ; 95 \%$ confidence interval, 0.46 to 1.48$)$. Conclusions: NHE training has the potential of increasing both knee flexors eccentric strength and biceps femoris long head fascicle length.
\end{abstract}

Keywords: posterior thigh, hamstring strength, hamstring strain injury, injury prevention

Muscle injuries have a high incidence in sports that involve high-speed movements ${ }^{1}$ such as track and field, ${ }^{2}$ rugby, ${ }^{3}$ basketball, ${ }^{4}$ soccer, ${ }^{5}$ and American football, ${ }^{6}$ as well as in ballet dancers. ${ }^{7}$ Muscle injuries are responsible for $10 \%$ to $55 \%$ of all injuries in sport, ${ }^{5,8-10}$ and they are common at the high school, ${ }^{11}$ college, ${ }^{12,13}$ and professional levels. ${ }^{5}$ About $92 \%$ of all lower limb muscle injuries affect hamstrings, adductors, quadriceps, or calf (gastrocnemius and/or soleus), and the hamstring muscles are the most commonly injuried. ${ }^{14}$ Most of hamstring strain injuries (HSI) are considered of moderate severity (54\%), with the athlete losing 17 days of practice on average. ${ }^{15}$ However, $15 \%$ of HSI are severe, which usually requires a longer rehabilitation period ( $>28$-d layoff). ${ }^{15}$ Furthermore, the reinjury rate of HSI is relatively high, especially when it affects the biceps femoris (18.4\%). ${ }^{16}$ Consequently, HSI impairs athletes' performance, ${ }^{17}$ and it has negative financial consequences for the club involved. ${ }^{18,19}$

With the aim of avoiding all the deleterious effects of an HSI, many studies ${ }^{20-26}$ have concentrated their efforts in identifying risk factors for HSI in an attempt to contribute to the development of prevention programs. The risk factors can be divided in nonmodifiable (eg, previous injuries, age, and genetics) and modifiable (eg, flexibility, fatigue, eccentric strength, and fascicle length)..$^{27,28}$ Knee flexors eccentric strength ${ }^{29-31}$ and biceps femoris fascicle length ${ }^{29,32,33}$ have received great attention in the last decade. For instance, a prospective cohort study ${ }^{29}$ evidenced that elite soccer players with knee flexors eccentric weakness $(<337 \mathrm{~N}$ assessed with the NordBord System ${ }^{34}$ ) and short biceps femoris long head

The authors are with the Graduate Program of Rehabilitation Sciences, Federal University of Health Sciences of Porto Alegre, Porto Alegre, Brazil. Medeiros (Diulian.medeiros@yahoo.com) is corresponding author. fascicle length $(<10.56 \mathrm{~cm})$ were 4.4 and 4.1 times more likely to sustain an HSI, respectively. Therefore, strategies capable of increasing both eccentric strength and fascicle length seem to be crucial to decrease risk of HSI.

Resistance training is conventionally performed with the same external load during the concentric and eccentric phases, but studies with different populations have demonstrated higher increments in muscle strength through eccentric overload training. ${ }^{35-38}$ Eccentric training also seems to generate greater strength gains than concentric training. ${ }^{39}$ Whereas concentric training presents small or nonsignificant impact on either muscle eccentric strength ${ }^{39}$ or fascicle length, ${ }^{40}$ training programs with eccentric overload seem to increase both eccentric strength and fascicle length. ${ }^{41,42}$ In fact, eccentric exercise has been suggested as an effective strategy to prevent HSI, ${ }^{20,24}$ and the Nordic hamstring exercise (NHE) is increasingly popular among athletes and coaching/ medical staffs. ${ }^{43,44}$

Studies have shown that NHE-based training has the potential to reduce HSI rate in athletes of baseball, ${ }^{25}$ rugby, ${ }^{3}$ and soccer, ${ }^{20,22,24,45}$ and this preventive effect of NHE is further supported by a recent meta-analysis ${ }^{46}$ which identified that NHE is capable of decreasing in 50\% the incidence of HSI. In face of the promising results of NHE as a preventive strategy against the HSI in athletes, clarifying the mechanisms responsible for the preventive effect of this resistance exercise with eccentric emphasis is pertinent. Hence, this systematic review and meta-analysis aims to analyze the effects of NHE on knee flexors eccentric strength and fascicle length.

\section{Evidence Acquisition}

The current study utilized PRISMA (Preferred Reporting Items for Systematic Review and Meta-analyses) guidelines for 
systematic reviews and meta-analysis. ${ }^{47}$ Prior to search, a review protocol was completed and registered at PROSPERO (CRD42018092699).

\section{Data Sources and Searches}

We searched the following electronic databases (from inception to April 2020): MEDLINE (accessed by PubMed), Physiotherapy Evidence Database (PEDro), and The Cochrane Central Register of Controlled Trials (Cochrane CENTRAL). In addition, we searched the references of published studies. Retrieved references were imported into EndNote X7 (Thomson Reuters, New York City, NY), where duplicates were subsequently deleted. The search comprised the following terms: "Hamstring Muscle," "Semitendinosus," "Semimembranosus," "Biceps Femoris," "Posterior thigh," "Muscle architecture," "Fascicle length," "Pennation angle," "Muscle thickness," "Nordic hamstring exercise," "Nordic curl," "Nordic curl exercise," and "Hamstring injury prevention," combined with a high-sensitivity combination of words used in the search for randomized clinical trials. ${ }^{48} \mathrm{We}$ included only publications in English. For the combination of the keywords, we utilized the Boolean terms "AND" and "OR."

\section{Eligibility Criteria}

We included randomized clinical trials and controlled clinical trials that evaluated the effects of NHE on eccentric strength and/or fascicle length. In order to improve the clarity of the information provided, the term "strength" will be used to refer the muscle ability to produce force. The following exclusion criteria were used: (1) samples comprised of people with any disease/dysfunction, (2) nonapplication of NHE, (3) nonevaluation of hamstring eccentric strength or fascicle length, (4) application of NHE in association with other exercises, (5) noninclusion of a control group (CG), (6) samples with mean age under 18 years old, and (7) studies with training protocol shorter than 4 weeks or 8 sessions. It should be mentioned that we chose to include participants of both genders because our analyses considered the difference between preintervention and postintervention whether than the peak values. Even though women might present lower peak torque when compared with men, ${ }^{49}$ the adaptations observed after training tend to be similar. As for fascicle length, a recent investigation has shown that fascicle length does not differ between sexes. ${ }^{50}$ Thus, the inclusion of both male and female participants is unlikely to affect our results.

\section{Studies Selection and Data Extraction}

Two investigators independently evaluated titles and abstracts of all articles identified by the search strategy. All abstracts that did not provide sufficient information regarding the inclusion and exclusion criteria were selected for full-text evaluation. In the second phase, the same reviewers independently evaluated the full-text articles and made their selection in accordance with the eligibility criteria. Disagreements between reviewers were solved by consensus. Using standardized forms, the same 2 reviewers independently conducted data extraction with regard to the methodological characteristics of the studies, number of participants, age, NHE training protocol, outcomes assessments, and results. Disagreements were also solved by consensus. The outcomes extracted were knee flexors eccentric strength (peak force or peak torque) and muscular fascicle length. It is important to highlight that the eccentric peak torque was evaluated by isokinetic dynamometry, while eccentric peak force was assessed during an NHE execution through specific devices with load cells.

\section{Quality Assessment}

The methodological quality of each study was independently assessed by 2 investigators, and any discrepancies were resolved by consensus. The quality and risk of bias were evaluated according to the Cochrane risk of bias tool, ${ }^{51}$ where 4 main domains of bias are assessed: selection bias, detection bias, attrition bias, and reporting bias. Studies without a clear description of these characteristics were considered unclear.

\section{Data Synthesis and Analysis}

Intervention effects for strength and fascicle length were calculated using standardized mean differences (SMDs) with 95\% confidence intervals (CIs), as all data were continuous. The mean change scores and SDs of the change scores from the intervention and CGs were used to calculate the SMD. If the SDs of the change scores were not reported, these were calculated using the formula, ${ }^{52}$ where correlation coefficients were conservatively set at $.5 .{ }^{53}$ A positive SMD represents an effect in favor of intervention group and a negative SMD an effect in favor of CG. Statistical heterogeneity of the treatment effects among studies was assessed using Cochran $Q$ test and the inconsistency $I^{2}$ test, in which values above $25 \%$ and $50 \%$ were considered indicative of moderate and high heterogeneity, respectively. ${ }^{54} \mathrm{~A}$ random effects model was selected for the analysis. All analyses were conducted using Review Manager (version 5.3; London, United Kingdom). For the studies that reported only standard error, we estimated the SD by multiplying the standard error by the square root of the sample size (n). For the studies that presented the values of torque in Newton meter per kilogram, ${ }^{55,56}$ we multiplied the values by the mean weight of the subjects in order to normalize the results and perform a more complete analysis. Furthermore, it is important to point out that for the study that presented its data only through graphs, ${ }^{57}$ the values were extracted using the Plot Digitizer. ${ }^{58}$ We explored heterogeneity between studies by rerunning the meta-analyses removing one paper at a time to check whether some individual study explained heterogeneity.

\section{Evidence Synthesis}

\section{Description of Studies}

The search strategy yielded 1932 articles, after the exclusion of the duplicates (560 studies) identified by the software Endnote; 1377 titles were analyzed, of which 29 studies were considered as potentially relevant and retrieved for detailed analysis. In the full-text analysis, 17 studies were excluded. Hence, 12 studies met the eligibility criteria and were included in the systematic review $(n=299)$, and 9 studies presented suitable data for metaanalysis $(n=205)$. Figure 1 shows the flow diagram of the studies included in this review, and Table 1 summarizes the studies' characteristics and their main results.

\section{Risk of Bias}

Of the studies included in this systematic review, $50 \%$ presented an adequate sequence generation, $25 \%$ reported allocation concealment; $58 \%$ had blinded assessment of outcomes, $58 \%$ described losses to follow-up and exclusions, and none of the included studies had incomplete outcome data or selectively reported the outcomes (Table 2). 

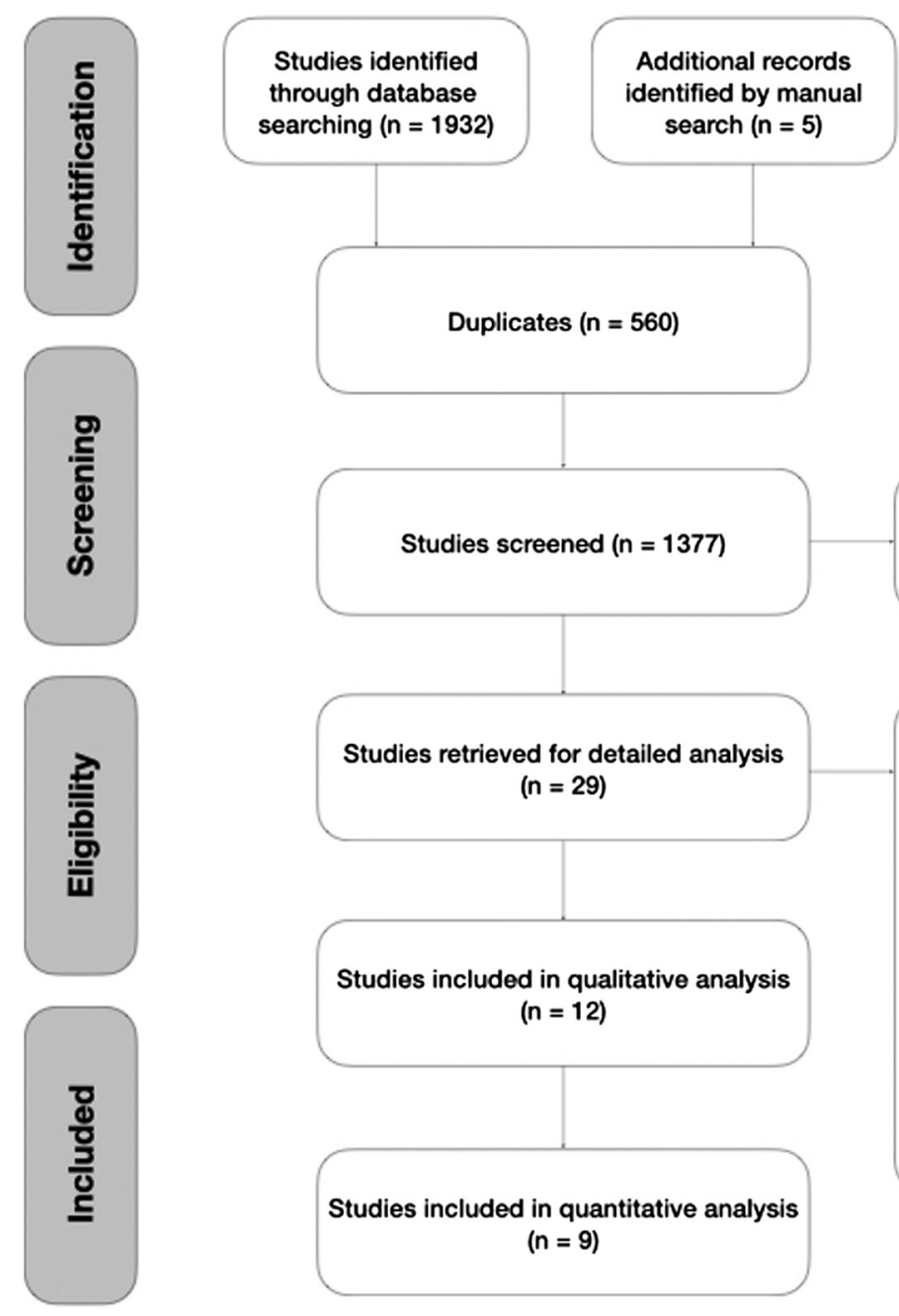

Studies excluded based on titles and/or abstract $(n=1348)$

17 full-text studies excluded:

- Multiple exercises training $(n=3)$

- injury prevention study $(n=5)$

- No NHE $(n=4)$

- Acute effect $(n=1)$

- No control/comparison group $(n=2)$

- Did not assess the variables of interest $(n=2)$

Figure 1 - Flowchart of the included studies. NHE indicates Nordic hamstring exercise.

\section{Effects of NHE on Eccentric Strength}

Eleven studies evaluated the effects of NHE on eccentric strength (peak torque or force). ${ }^{56,57,59-63,65-68}$ Eight studies presented suitable data for meta-analysis. Analysis of studies that evaluated eccentric peak torque $55,56,59,61,66$ showed a significant difference between NHE and CG $\left(0.68 ; 95 \%\right.$ CI, 0.29 to $1.06 ; I^{2}: 0 \%$; Figure 2). Analysis of studies that assessed eccentric peak force $^{57,62,67}$ also showed a significant difference between NHE and CG $\left(01.11 ; 95 \%\right.$ CI, 0.62 to $1.61: I^{2}: 55 \%$; Figure 3$)$. The high heterogeneity in the latter analysis can be explained by the study from Bourne et $\mathrm{al}^{57}$ that worked with a different population when compared with the studies from Ishøi et $\mathrm{al}^{62}$ and Suarez-Arrones et al. ${ }^{67}$ It is reasonable to assume that the participants' physical conditioning differences (active male vs soccer players) may have played a role in the difference in the adaptations found. When the study from Bourne et $\mathrm{al}^{57}$ was excluded from the meta-analysis, the heterogeneity was $0 \%\left(0.88 ; 95 \% \mathrm{CI}, 0.33\right.$ to $\left.1.42: I^{2}: 0 \%\right)$.

\section{Effects of NHE on Fascicle Length}

Five studies ${ }^{56,57,63,64,66}$ evaluated the effects of NHE on biceps femoris long head fascicle length. All of them compared NHE with a CG (no intervention or alternative exercise). Four studies ${ }^{56,57,64,66}$ provided suitable data for meta-analysis. The analysis showed that
NHE is effective in increasing fascicle length when compared with a CG $\left(0.97 ; 95 \%\right.$ CI, 0.46 to $1.48: I^{2}: 71 \%$; Figure 4$)$. The high heterogeneity in the analysis of fascicle length can once again be explained by the study from Bourne et al. ${ }^{57}$ The authors performed a longer NHE training (10 wk) when compared with the other studies $\left(4^{66}\right.$ and $\left.6 \mathrm{wk}^{56,64}\right)$. It is possible that the longer training period might have contributed to the higher increase in fascicle length observed by Bourne et al. ${ }^{57}$ When this study was excluded from the meta-analysis, the heterogeneity was $0 \%(0.65 ; 95 \% \mathrm{CI}$, 0.09 to $1.20: I^{2}: 0 \%$ ).

\section{Discussion}

\section{Summary of Evidence}

The evidence presented in the current review showed that NHEbased training has the potential to enhance both HSI risk factors assessed in this review: knee flexors eccentric strength and biceps femoris long head fascicle length.

\section{Effects of NHE on Strength}

Hamstring muscles have a fundamental role on sprinting, especially during the terminal swing phase, when there is an intense 


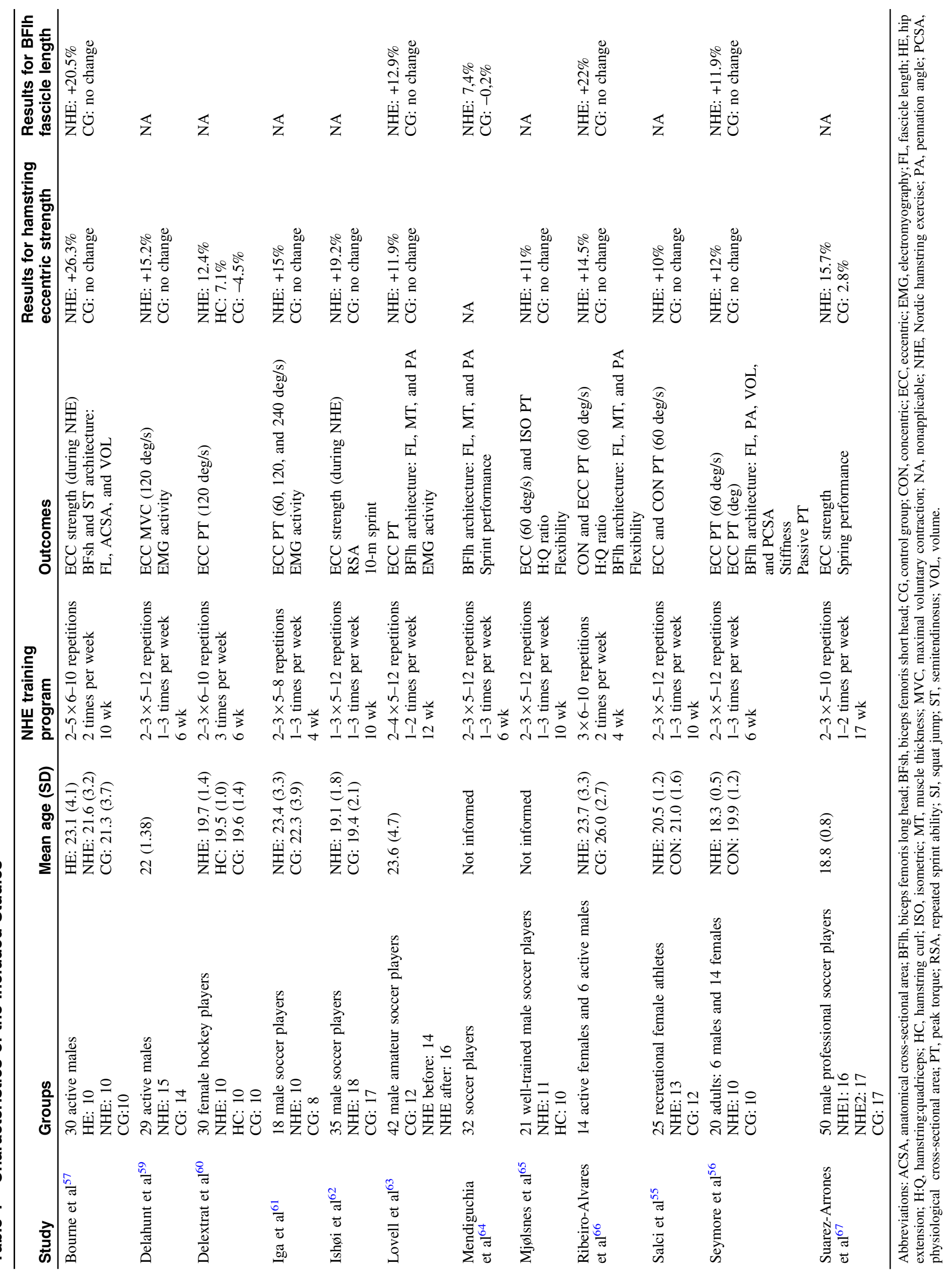


Table 2 Risk of Bias of the Included Studies

\begin{tabular}{|c|c|c|c|c|c|c|}
\hline Study & $\begin{array}{l}\text { Adequate } \\
\text { sequence } \\
\text { generation }\end{array}$ & $\begin{array}{l}\text { Allocation } \\
\text { concealment }\end{array}$ & $\begin{array}{l}\text { Blinding } \\
\text { of outcome } \\
\text { assessors }\end{array}$ & $\begin{array}{l}\text { Description } \\
\text { of losses and } \\
\text { exclusions }\end{array}$ & $\begin{array}{l}\text { Incomplete } \\
\text { outcome } \\
\text { data }\end{array}$ & $\begin{array}{l}\text { Selective } \\
\text { outcome } \\
\text { reporting }\end{array}$ \\
\hline Bourne et al ${ }^{57}$ & Low risk & Unclear & Low risk & Unclear & Low risk & Low risk \\
\hline Delahunt et al ${ }^{59}$ & Low risk & Low risk & Low risk & Unclear & Low risk & Low risk \\
\hline Delextrat et $\mathrm{al}^{60}$ & Low risk & Unclear & Low risk & Low risk & Low risk & Low risk \\
\hline Iga et $\mathrm{al}^{61}$ & Unclear & Unclear & Low risk & Unclear & Low risk & Low risk \\
\hline Ishøi et $\mathrm{al}^{62}$ & Low risk & Low risk & Low risk & Low risk & Low risk & Low risk \\
\hline Lovell et $\mathrm{al}^{63}$ & Unclear & Unclear & Unclear & Low risk & Low risk & Low risk \\
\hline Mendiguchia et $\mathrm{al}^{64}$ & Unclear & Unclear & Low risk & Low risk & Low risk & Low risk \\
\hline Mjølsnes et $\mathrm{al}^{65}$ & Low risk & Unclear & Low risk & Low risk & Low risk & High risk \\
\hline Ribeiro-Alvares et al ${ }^{66}$ & Low risk & Unclear & High risk & Unclear & Low risk & Low risk \\
\hline Salci et $\mathrm{al}^{55}$ & Unclear & Low risk & Unclear & Low risk & Low risk & Low risk \\
\hline Seymore et $\mathrm{al}^{56}$ & Unclear & Unclear & Unclear & Unclear & Low risk & Low risk \\
\hline Suarez-Arrones et al ${ }^{67}$ & High risk & High risk & Unclear & Low risk & Low risk & Low risk \\
\hline
\end{tabular}

\begin{tabular}{|c|c|c|c|c|c|c|c|c|c|c|c|c|}
\hline Study or subgroup & \multicolumn{3}{|c|}{ NHE } & \multicolumn{3}{|c|}{ CG } & \multicolumn{2}{|r|}{ Standard mean difference } & \multicolumn{3}{|c|}{$\begin{array}{l}\text { Standard mean difference } \\
\text { IV, Random, } 95 \% \mathrm{CI}\end{array}$} & \\
\hline Delahunt et al ${ }^{59}$ & 27 & 29.28 & 15 & -1.1 & 28.91 & 14 & $24.7 \%$ & 0.94 (0.17 to 1.71$)$ & & & 二- & \\
\hline Iga et al ${ }^{61}$ & 17 & 42.5 & 10 & -13 & 37.5 & 8 & $15.8 \%$ & $0.71(-0.26$ to 1.67$)$ & & & & \\
\hline Ribeiro-Alvares et al 66 & 16 & 29.27 & 10 & 1.5 & 30.25 & 10 & $18.6 \%$ & $0.47(-0.42$ to 1.36$)$ & & & & \\
\hline Salci et al ${ }^{55}$ & 12.7 & 14.69 & 13 & 1.12 & 15.54 & 12 & $22.2 \%$ & $0.74(-0.07$ to 1.56$)$ & & & -1 & \\
\hline Seymore et al ${ }^{56}$ & 12.83 & 50.86 & 10 & -5.13 & 21.08 & 10 & $18.7 \%$ & $0.44(-0.45$ to 1.33$)$ & & & & \\
\hline Total $(95 \% \mathrm{Cl})$ & & & 58 & & & 54 & $100.0 \%$ & 0.68 (0.29 to 1.06$)$ & & & & \\
\hline $\begin{array}{l}\text { Heterogeneity: } \tau^{2}=.00 \\
\text { Test for overall effect: }\end{array}$ & $\begin{array}{l}0.95, \mathrm{~d} \\
6(P=.0\end{array}$ & $\begin{array}{l}\mathrm{f}=4(P \\
0005)\end{array}$ & $=.92)$ & $; I^{2}=0 \%$ & & & & & -4 & $\begin{array}{l}1 \\
-2\end{array}$ & NHE & \\
\hline
\end{tabular}

Figure 2 - Analysis of eccentric strength during isokinetic dynamometry. CG indicates control group; CI, confidence interval; NHE, Nordic hamstring exercise.

\begin{tabular}{|c|c|c|c|c|c|c|c|c|c|c|c|}
\hline Study or subgroup & \multicolumn{3}{|c|}{ NHE } & \multicolumn{3}{|c|}{ CG } & \multicolumn{2}{|r|}{ Standard mean difference } & \multicolumn{2}{|c|}{$\begin{array}{l}\text { Standard mean difference } \\
\text { IV, Fixed, } 95 \% \mathrm{Cl}\end{array}$} & \\
\hline Bourne et al ${ }^{57}$ & 97.79 & 62.21 & 10 & -9.63 & 24.16 & 10 & $18.2 \%$ & 2.18 (1.02 to 3.34$)$ & & - - & \\
\hline Ishoi et al ${ }^{62}$ & 61.7 & 61.63 & 11 & -9.3 & 66.93 & 14 & $33.5 \%$ & $1.06(0.21$ to 1.91$)$ & & $-1-$ & \\
\hline Suares-Arrones et al ${ }^{67}$ & 88.5 & 100.4 & 16 & 0.9 & 125.1 & 17 & $48.3 \%$ & 0.75 (0.04 to 1.46$)$ & & F- & \\
\hline Total $(95 \% \mathrm{Cl})$ & & & 37 & & & 41 & $100.0 \%$ & $1.11(0.62$ to 1.61$)$ & & & \\
\hline \multicolumn{4}{|c|}{$\begin{array}{l}\text { Heterogeneity: } \chi^{2}=4.29, \mathrm{df}=2(P=.12) ; I^{2}=53 \% \\
\text { Test for overall effect: } Z=4.43(P<.00001)\end{array}$} & & & & & -10 & -5 & ${ }^{+}{ }_{\mathrm{NHE}}$ & 10 \\
\hline
\end{tabular}

Figure 3 - Analysis of eccentric strength during Nordic hamstring exercise test. CG indicates control group; CI, confidence interval; NHE, Nordic hamstring exercise.

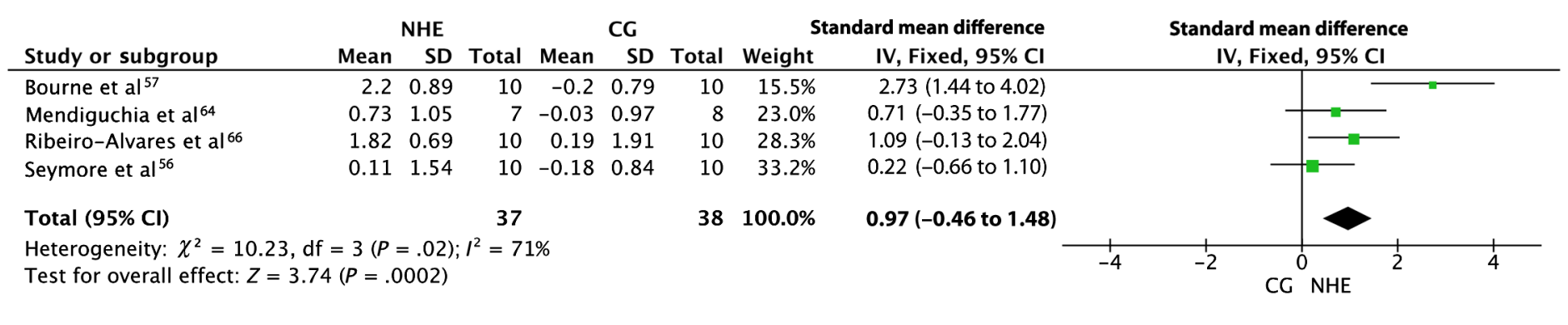

Figure 4 - Analysis of fascicle length. CG indicates control group; CI, confidence interval; NHE, Nordic hamstring exercise. 
active lengthening muscle action to decelerate both the hip flexion and the knee extension. ${ }^{69,70}$ Therefore, it seems plausible that increasing eccentric strength might decrease the risk of sustaining an HSI. However, the literature is not definitive regarding the role of poor hamstring strength as a risk factor for HSI. For instance, Van Dyk et al $^{71}$ concluded that poor hamstring strength is a weak risk factor for HSI after assessing 614 professional soccer players through isokinetic dynamometry at preseason and following them along the season. On the other hand, Timmins et $\mathrm{al}^{29}$ and Lee et $\mathrm{al}^{30}$ prospectively assessed the hamstring eccentric strength of professional soccer players during the NHE execution and isokinetic dynamometry, respectively; those studies found that injured players throughout the subsequent season were approximately $13 \%$ to $19 \%$ weaker than the uninjured players. Despite these conflicting findings, it seems reasonable to assume that muscle weakness can never be considered as normal, and should always be addressed properly, especially in athletic populations.

The current review evidenced that NHE-based training was associated with increase in knee flexors eccentric strength. It is important to highlight that NHE training generated significant increases on eccentric strength in all studies assessed by our review, regardless the type of evaluation performed. When tests were performed on the isokinetic dynamometer, strength gains ranged between $10 \%$ and $15 \%$, while studies comprising tests on the NHE device found increases from $16 \%$ to $26 \%$. The greater percentage strength gains reported on the NHE device is probably related to training specificity. ${ }^{72}$ As the NHE device mimics the exact movement performed during the training program, it is reasonable to assume that the gains would be higher when compared with the isokinetic dynamometry, which involves a totally different testing setting.

Studies included in this review assessed participants with different conditioning levels (from physically active university students $^{66}$ to professional soccer players ${ }^{62}$ ). It is a factor that significantly influences the responses to any type of strength training, including the NHE. In addition, the training protocols varied among the studies regarding training period, weekly frequency, and total training volume. NHE training volume has been an issue of current interest as it remains a challenge for coaches and conditioning trainers to fit NHE training in an already busy schedule. ${ }^{73}$ There seems to be a concern that the NHE added to the athletes' training routine will promote an overload of muscular work. Thus, the dose of NHE training that is sufficient to generate muscle adaptations without promoting excessive levels of fatigue remains uncertain.

Severo-Silveira et al $^{74}$ compared the effects of 2 NHE training programs (progressive workload, 236 repetitions along $8 \mathrm{wk}$; and constant workload, 138 repetitions along 8 wk) in Rugby players, and found that only the group trained with the greater training volume increased knee flexors eccentric strength. Nevertheless, performing NHE progressively during the season might not be suitable for the athletes' training routines. A possible solution for this issue could be performing high-volume training followed by a low-volume training. Presland et al ${ }^{75}$ reported that 2 weeks of highvolume NHE training followed by 4 weeks of low-volume training (128 repetitions along $6 \mathrm{wk}$ ) generate similar adaptations when compared with 6 weeks of high-volume training (440 repetitions along $6 \mathrm{wk}$ ). Therefore, performing a high-volume training during the preseason and maintaining the gains with a low-volume training during the season might be more suitable for the teams' training routines. However, it is important to mention that there is no consensus in current literature regarding the minimal NHE training volume required to promote significant increase in strength. For instance, all studies included in this review reported increase in hamstring eccentric strength, regardless of the periodization employed. This highlights the need for further studies regarding muscular adaptations promoted by the NHE training, especially to establish the optimal training volume for athletic populations.

\section{Effects of NHE on Fascicle Length}

In the last decades, the study of muscle architecture has been enabled by the introduction of $2 \mathrm{D}$ image ultrasound in this field. ${ }^{76}$ This cost- and time-effective noninvasive and easily accessible tools have helped to expand the assessment of muscle thickness, pennation angle, and fascicle length of a range of muscles. Biceps femoris long head fascicle length is the most remarkable muscle architecture outcome related to HSI. Evidence suggests that previously injured biceps femoris long head muscles present significantly shorter fascicles than muscles without history of injury, ${ }^{77}$ which might be one of the reasons why previously injured muscles have an increased chance of sustaining a new injury. ${ }^{3}$ Even more impressive are prospective findings that biceps femoris long head fascicles shorter than $10.56 \mathrm{~cm}$ at preseason increase more than 4 times the risk to HSI along the season in professional soccer players. ${ }^{29}$ Therefore, biceps femoris long head fascicle length is considered the newest and a very promising risk factor for HSI in sports.

Fascicle length increase changes the force-velocity and forcelength relationships, which directly impacts muscle function. ${ }^{78}$ In theory, a muscle with longer fascicles contains a higher amount of in-series aligned sarcomeres, which would increase muscle contraction velocity ${ }^{79}$ and also prevent the muscle from damage due to over-lengthening. ${ }^{32}$ On the other hand, a muscle with reduced fascicle length presents an increased muscle susceptibility to eccentrically induced microscopic muscle damage, which could facilitate a macroscopic damage. ${ }^{80}$ Hence, exercises capable of increasing fascicle length might contribute to prevent HSI.

Our results support NHE training as an effective strategy to increase the biceps femoris long head fascicle length, as previously evidenced through isokinetic eccentric training of the hamstrings ${ }^{40}$ and other muscle groups. ${ }^{42,81}$ All studies included in the current review that addressed fascicle length found that NHE training promoted significant increases in that outcome, independently of the training periodization or participants conditioning status. It is worth pointing out in this context that the only 2 studies $^{74,75}$ to this date that have compared low and high volume of NHE training found that fascicle length is increased even with low-volume training programs. This information is relevant for clubs that have difficulties implementing high volumes of NHE training due to their tight schedule.

Interestingly, fascicle length enhancement seems to occur since the first month of NHE training. ${ }^{66}$ Short-term responses might be useful for athletes who have short periods of preseason such as soccer players, ${ }^{43,44}$ given that they could start the competitive season with longer fascicles and less susceptibility to HSI. However, the quick fascicle length adaptation in response to NHE training has been evidenced only in a nonathletic population. ${ }^{66}$ In well-trained athletes, a longer training period (8-wk NHE training program) seems to be necessary to increase the hamstring fascicle length. ${ }^{74}$ Nonetheless, further investigations are needed to verify the short-term responses of fascicle length in this population. 
According to the logistic regression performed by Timmins et al, ${ }^{29}$ an increase in only $11 \%$ in biceps femoris long head fascicle length enables a decrease of approximately $21 \%$ in the probability of HSI. On the other hand, the same study demonstrated that increments of at least $50 \%$ in hamstring eccentric strength are required to achieve a similar decrease in the probability of injury, ${ }^{29}$ which is a muscle strengthening hardly achieved in well-trained athletes. In the present review, the studies found increases in fascicle length from $12 \%$ to $22 \%$, while the increases in eccentric strength ranged between $10 \%$ and $26 \%$. Therefore, it seems reasonable to hypothesize that fascicle length changes might have a key role for the preventive effect of NHE. However, it is important to point out that fascicle length and eccentric strength only contribute to preventing HSI to some point. There are several other determinants suggested by prospective studies (eg, lumbopelvic stability, posterior chain flexibility, workload) that play a role in HSI and will be not modified through NHE training.

\section{Strengths and Limitations}

The current review is not without limitations. The main limitation of the present review is that we were not able to gather the totality of the studies in the quantitative analysis. Another limitation is the low to moderate methodological quality of the included studies. However, a strategy for a sensitive and comprehensive search to assure the location of all most recent studies in this field was held, further highlighting the need for additional investigations in that field. To the best of our knowledge, the current study is the first meta-analysis to exclusively analyze the role of NHE on specific hamstring injury risk factors (eccentric strength and fascicle length). Previous reviews ${ }^{82,83}$ have focused on HSI incidence, and even though we agree that this is the main variable in any injury prevention protocol, it is important to elucidate the mechanisms that underpin any positive results.

\section{Conclusions}

The present review evidenced that NHE is a valid strategy to enhance both knee flexors eccentric strength and fascicle length, 2 evidence-based risk factors for HSI. Our findings elucidated 2 of the main mechanisms that explain, at least in part, the preventive effect of NHE against HSI, and reinforce the adoption of this exercise during both training and rehabilitation programs. Nevertheless, further research is needed to verify the best NHE training protocol to modify eccentric strength and fascicle length without impairing athletes' performance and increasing risk of HSI.

\section{Acknowledgments}

D.M.M. thanks CAPES-Brazil for the scholarship. B.M.B. thanks CNPqBrazil for the research productivity fellowship. This work was not funded by any external source.

\section{References}

1. Brukner P. Hamstring injuries: prevention and treatment—an update. Br J Sports Med. 2015;49(19):1241-1244. doi:10.1136/bjsports2014-094427

2. Malliaropoulos N, Isinkaye T, Tsitas K, Maffulli N. Reinjury after acute posterior thigh muscle injuries in elite track and field athletes.
Am J Sports Med. 2011;39(2):304-310. PubMed ID: 21051422 doi: 10.1177/0363546510382857

3. Brooks JHM, Fuller CW, Kemp SPT, Reddin DB. Incidence, risk, and prevention of hamstring muscle injuries in professional rugby union. Am J Sport Med. 2006;34(8):1297-1306. doi:10.1177/ 0363546505286022

4. Borowski LA, Yard EE, Fields SK, Comstock RD. The Epidemiology of US High School Basketball Injuries, 2005-2007. Am J Sports Med. 2008;36(12):2328-2335. PubMed ID: 18765675 doi:10.1177/ 0363546508322893

5. Ekstrand J, Hägglund M, Waldén M. Epidemiology of muscle injuries in professional football (soccer). Am J Sports Med. 2011;39(6): 1226-1232. PubMed ID: 21335353 doi:10.1177/0363546510395879

6. Feeley BT, Kennelly S, Barnes RP, et al. Epidemiology of National Football League Training Camp Injuries from 1998 to 2007. Am J Sports Med. 2008;36(8):1597-1603. PubMed ID: 18443276 doi: $10.1177 / 0363546508316021$

7. Askling CM, Tengvar M, Saartok T, Thorstensson A. Proximal hamstring strains of stretching type in different sports: injury situations, clinical and magnetic resonance imaging characteristics, and return to sport. Am J Sports Med. 2008;36(9):1799-1804. PubMed ID: 18448581 doi:10.1177/0363546508315892

8. Garrett WE. Muscle strain injuries. Am J Sports Med. 1996;24(6): S2-S8. doi:10.1177/036354659602406S02

9. Huard J, Li Y, Fu FH. Muscle injuries and repair: current trends in research. J Bone Joint Surg Am. 2002;84(5):822-832. doi:10.2106/ 00004623-200205000-00022

10. Järvinen TAH, Järvinen TLN, Kääriäinen M, Kalimo H, Järvinen M. Muscle injuries: biology and treatment. Am J Sports Med. 2005; 33(5):745-764. PubMed ID: 15851777 doi:10.1177/03635465052 74714

11. Shankar PR, Fields SK, Collins CL, Dick RW, Comstock RD. Epidemiology of High School and Collegiate Football Injuries in the United States, 2005-2006. Am J Sports Med. 2007;35(8):1295-1303. PubMed ID: 17369559 doi:10.1177/0363546507299745

12. Price RJ, Hawkins RD, Hulse MA, Hodson A. The Football Association medical research programme: an audit of injuries in academy youth football. Br J Sports Med. 2004;38(4):466-471. PubMed ID: 15273188 doi:10.1136/bjsm.2003.005165

13. Marshall SW, Hamstra-Wright KL, Dick R, Grove KA, Agel J. Descriptive epidemiology of collegiate women's softball injuries: National Collegiate Athletic Association injury surveillance system, 1988-1989 through 2003-2004. J Athl Train. 2007;42(2):286-294. doi:10.1016/S0276-1092(08)79204-6

14. Ekstrand J, Hägglund M, Waldén M. Injury incidence and injury patterns in professional football: the UEFA injury study. Br J Sports Med. 2011;45(7):553-558. doi:10.1136/bjsm.2009.060582

15. Ekstrand J, Waldén M, Hägglund M. Hamstring injuries have increased by $4 \%$ annually in men's professional football, since 2001: a 13-year longitudinal analysis of the UEFA Elite Club injury study. Br J Sports Med. 2016;50(12):731-737. PubMed ID: 26746908 doi:10.1136/bjsports-2015-095359

16. Hallén A, Ekstrand J. Return to play following muscle injuries in professional footballers. J Sports Sci. 2014;32(13):1229-1236. PubMed ID: 24784885 doi:10.1080/02640414.2014.905695

17. Tol JL, Hamilton B, Eirale C, Muxart P, Jacobsen P, Whiteley R. At return to play following hamstring injury the majority of professional football players have residual isokinetic deficits. Br J Sports Med. 2014;48(18):1364-1369. PubMed ID: 24493666 doi:10.1136/ bjsports-2013-093016

18. Woods C. The football association medical research programme: an audit of injuries in professional football—analysis of hamstring 
injuries. Br J Sports Med. 2004;38(1):36-41. PubMed ID: 14751943 doi:10.1136/bjsm.2002.002352

19. Hägglund M, Waldén M, Magnusson $H$, Kristenson K, Bengtsson $H$, Ekstrand J. Injuries affect team performance negatively in professional football: an 11-year follow-up of the UEFA Champions League injury study. Br J Sports Med. 2013;47(12):738-742. PubMed ID: 23645832 doi:10.1136/bjsports-2013-092215

20. Petersen J, Thorborg K, Nielsen MB, Budtz-Jørgensen E, Hölmich P. Preventive effect of eccentric training on acute hamstring injuries in men's soccer: a cluster-randomized controlled trial. Am J Sports Med. 2011;39(11):2296-2303. PubMed ID: 21825112 doi:10.1177/ 0363546511419277

21. Schache A. Eccentric hamstring muscle training can prevent hamstring injuries in soccer players. J Physiother. 2012;58(1):58. PubMed ID: 22341384 doi:10.1016/S1836-9553(12)70074-7

22. van der Horst N, Smits D-W, Petersen J, Goedhart EA, Backx FJG. The preventive effect of the Nordic hamstring exercise on hamstring injuries in amateur soccer players. Am J Sports Med. 2015;43(6): 1316-1323. doi:10.1177/0363546515574057

23. Nichols AW. Does eccentric training of hamstring muscles reduce acute injuries in soccer? Clin J Sport Med. 2013;23(1):85-86. PubMed ID: 23269328 doi:10.1097/JSM.0b013e31827e9f40

24. Arnason A, Andersen TE, Holme I, Engebretsen L, Bahr R. Prevention of hamstring strains in elite soccer: an intervention study. Scand J Med Sci Sport. 2008;18(1):40-48. doi:10.1111/j.1600-0838.2006. 00634.x

25. Seagrave RA, Perez L, McQueeney S, Toby EB, Key V, Nelson JD. Preventive effects of eccentric training on acute hamstring muscle injury in professional baseball. Orthop J Sport Med. 2014;2(6): 232596711453535. doi:10.1177/2325967114535351

26. Askling C, Karlsson J, Thorstensson A. Hamstring injury occurrence in elite soccer players after preseason strength training with eccentric overload. Scand J Med Sci Sport. 2003;13(4):244-250. doi:10.1034/ j.1600-0838.2003.00312.x

27. Opar DA, Williams MD, Shield AJ. Hamstring strain injuries: factors that lead to injury and re-injury. Sport Med.2012;42(3):209-226. doi: 10.2165/11594800-000000000-00000

28. Larruskain J, Celorrio D, Barrio I, et al. Genetic variants and hamstring injury in soccer: an association and validation study. Med Sci Sports Exerc. 2018;50(2):361-368. PubMed ID: 28976491 doi:10. 1249/MSS.0000000000001434

29. Timmins RG, Bourne MN, Shield AJ, Williams MD, Lorenzen C, Opar DA. Short biceps femoris fascicles and eccentric knee flexor weakness increase the risk of hamstring injury in elite football (soccer): a prospective cohort study. Br J Sports Med. 2016;50(24):1524-1535. PubMed ID: 26675089 doi:10.1136/bjsports-2015-095362

30. Lee JWY, Mok KM, Chan HCK, Yung PSH, Chan KM. Eccentric hamstring strength deficit and poor hamstring-to-quadriceps ratio are risk factors for hamstring strain injury in football: a prospective study of 146 professional players. J Sci Med Sport. 2018;21(8):789-793. PubMed ID: 29233665 doi:10.1016/j.jsams.2017.11.017

31. Bourne MN, Opar DA, Williams MD, Shield AJ. Eccentric knee flexor strength and risk of hamstring injuries in rugby union. Am J Sports Med. 2015;43(11):2663-2670. PubMed ID: 26337245 doi: $10.1177 / 0363546515599633$

32. Timmins RG, Shield AJ, Williams MD, Lorenzen C, Opar DA. Architectural adaptations of muscle to training and injury: a narrative review outlining the contributions by fascicle length, pennation angle and muscle thickness. Br J Sports Med. 2016;50(23):1467-1472. PubMed ID: 26817705 doi:10.1136/bjsports-2015-094881

33. Timmins RG, Bourne MN, Hickey JT, et al. Effect of prior injury on changes to biceps femoris architecture across an Australian Football
League season. Med Sci Sports Exerc. 2017;49(10):2102-2109. PubMed ID: 28548976 doi:10.1249/MSS.0000000000001333

34. Opar DA, Piatkowski T, Williams MD, Shield AJ. A novel device using the Nordic hamstring exercise to assess eccentric knee flexor strength: a reliability and retrospective injury study. J Orthop Sports Phys Ther. 2013;43(9):636-640. PubMed ID: 23886674 doi:10. 2519/jospt.2013.4837

35. Onambélé GL, Maganaris CN, Mian OS, et al. Neuromuscular and balance responses to flywheel inertial versus weight training in older persons. J Biomech. 2008;42(7):956. doi:10.1016/j.jbiomech.2008. 09.004

36. Walker S, Blazevich AJ, Haff GG, Tufano JJ, Newton RU, Häkkinen K. Greater strength gains after training with accentuated eccentric than traditional isoinertial loads in already strength-trained men. Front Physiol. 2016;27(7):149. doi:10.3389/fphys.2016.00149

37. Friedmann-Bette B, Bauer T, Kinscherf R, et al. Effects of strength training with eccentric overload on muscle adaptation in male athletes. Eur J Appl Physiol. 2010;108(4):821-836. doi:10.1007/s00421-0091292-2

38. Gerber JP, Marcus RL, Dibble LE, Greis PE, Burks RT, LaStayo PC. Effects of early progressive eccentric exercise on muscle size and function after anterior cruciate ligament reconstruction: a 1-year follow-up study of a randomized clinical trial. Phys Ther. 2009;89(1): 51-59. PubMed ID: 18988664 doi:10.2522/ptj.20070189

39. Roig M, O'Brien K, Kirk G, et al. The effects of eccentric versus concentric resistance training on muscle strength and mass in healthy adults: a systematic review with meta-analysis. Br J Sports Med. 2009; 43(8):556-568. PubMed ID: 18981046 doi:10.1136/bjsm.2008. 051417

40. Timmins RG, Ruddy JD, Presland J, et al. Architectural changes of the biceps femoris long head after concentric or eccentric training. Med Sci Sports Exerc. 2016;48(3):499-508. PubMed ID: 26460634 doi:10.1249/MSS.0000000000000795

41. Geremia JM, Baroni BM, Bobbert MF, Bini RR, Lanferdini FJ, Vaz MA. Effects of high loading by eccentric triceps surae training on Achilles tendon properties in humans. Eur J Appl Physiol. 2018; 118(8):1725-1736. PubMed ID: 29858689 doi:10.1007/s00421018-3904-1

42. Baroni BM, Rodrigues R, Franke RA, Geremia JM, Rassier DE, Vaz MA. Time course of neuromuscular adaptations to knee extensor eccentric training. Int J Sports Med. 2013;34(10):904-911. PubMed ID: 23526592 doi:10.1055/s-0032-1333263

43. McCall A, Carling C, Nedelec M, et al. Risk factors, testing and preventative strategies for non-contact injuries in professional football: current perceptions and practices of 44 teams from various premier leagues. Br J Sports Med. 2014;48(18):1352-1357. PubMed ID: 24837243 doi:10.1136/bjsports-2014-093439

44. Meurer MC, Silva MF, Baroni BM. Strategies for injury prevention in Brazilian football: perceptions of physiotherapists and practices of premier league teams. Phys Ther Sport. 2017;28:1-8. PubMed ID: 28886473 doi:10.1016/j.ptsp.2017.07.004

45. Gabbe BJ, Branson R, Bennell KL. A pilot randomised controlled trial of eccentric exercise to prevent hamstring injuries in communitylevel Australian Football. J Sci Med Sport. 2006;9(1-2):103-109. PubMed ID: 16574482 doi:10.1016/j.jsams.2006.02.001

46. van Dyk N, Behan FP, Whiteley R. Including the Nordic hamstring exercise in injury prevention programmes halves the rate of hamstring injuries: a systematic review and meta-analysis of 8459 athletes. $\mathrm{Br}$ J Sport Med. 2019;53(21):1362-1370. doi:10.1136/bjsports-2018100045

47. Shamseer L, Moher D, Clarke M, et al. Preferred reporting items for systematic review and meta-analysis protocols (PRISMA-P) 2015: 
elaboration and explanation. BMJ. 2015;349:g7647. doi:10.1136/bmj. g7647

48. Robinson KA, Dickersin K. Development of a highly sensitive search strategy for the retrieval of reports of controlled trials using PubMed. Int J Epidemiol. 2002;31(1):150-153. PubMed ID: 11914311 doi: 10.1093/ije/31.1.150

49. Hewett TE, Myer GD, Zazulak BT. Hamstrings to quadriceps peak torque ratios diverge between sexes with increasing isokinetic angular velocity. J Sci Med Sport. 2008;11(5):452-459. PubMed ID: 17875402 doi:10.1016/j.jsams.2007.04.009

50. Behan FP, Moody R, Patel TS, Lattimore E, Maden-Wilkinson TM, Balshaw TG. Biceps femoris long head muscle fascicle length does not differ between sexes. J Sports Sci. 2019;37(21):2452-2458. PubMed ID: 31303128 doi:10.1080/02640414.2019.1641016

51. Higgins JPT, Altman DG. Higgins 2011. In: Higgins JPT, Green S, eds. Cochrane Handbook for Systematic Reviews of Interventions. Version 5.1.0 [Updated March 2011]. Chichester, United Kingdom: The Cochrane Collaboration; 2011. doi:10.1002/ 9780470712184.ch8

52. Higgins JP, Green S. Cochrane Handbook for Systematic Reviews of Interventions: Cochrane Book Series. Chichester, United Kingdom: The Cochrane Collaboration; 2008. doi:10.1002/ 9780470712184

53. Follmann D, Elliott P, Suh I, Cutler J. Variance imputation for overviews of clinical trials with continuous response. J Clin Epidemiol. 1992;45(7):769-773. PubMed ID: 1619456 doi:10.1016/08954356(92)90054-Q

54. Higgins JPT, Thompson SG, Deeks JJ, Altman DG. Measuring inconsistency in meta-analyses. BMJ Br Med J. 2003;327(7414): 557-560. doi:10.1136/bmj.327.7414.557

55. Salci Y, Yildirim A, Celik O, Ak E, Kocak S, Korkusuz F. The effects of eccentric hamstring training on lower extremity strength and landing kinetics in recreational female athletes. Isokinet Exerc Sci. 2013;21(1):11-18. doi:10.3233/IES-2012-0466

56. Seymore KD, Domire ZJ, DeVita P, Rider PM, Kulas AS. The effect of Nordic hamstring strength training on muscle architecture, stiffness, and strength. Eur J Appl Physiol. 2017;117(5):943-953. PubMed ID: 28280975 doi:10.1007/s00421-017-3583-3

57. Bourne MN, Duhig SJ, Timmins RG, et al. Impact of the Nordic hamstring and hip extension exercises on hamstring architecture and morphology: implications for injury prevention. Br J Sports Med. 2017;51(5):469-477. PubMed ID: 27660368 doi:10.1136/bjsports2016-096130

58. Jelicic Kadic A, Vucic K, Dosenovic S, Sapunar D, Puljak L. Extracting data from figures with software was faster, with higher interrater reliability than manual extraction. J Clin Epidemiol. 2016; 74:119-123. doi:10.1016/j.jclinepi.2016.01.002

59. Delahunt E, McGroarty M, De Vito G, Ditroilo M. Nordic hamstring exercise training alters knee joint kinematics and hamstring activation patterns in young men. Eur J Appl Physiol. 2016; 116(4):663-672. PubMed ID: 26754149 doi:10.1007/s00421-0153325-3

60. Delextrat A, Bateman J, Ross C, et al. Changes in torque-angle profiles of the hamstrings and hamstrings-to-quadriceps ratio after two hamstring strengthening exercise interventions in female hockey players. J Strength Cond Res. 2020;34(2):396-405. PubMed ID: 31425454 doi:10.1519/JSC.0000000000003309

61. Iga J, Fruer CS, Deighan M, Croix MDS, James DVB. Nordic hamstrings exercise-engagement characteristics and training responses. Int J Sports Med. 2012;33(12):1000-1004. PubMed ID: 22895870 doi:10.1055/s-0032-1304591
62. Ishøi L, Hölmich P, Aagaard P, Thorborg K, Bandholm T, Serner A. Effects of the Nordic hamstring exercise on sprint capacity in male football players: a randomized controlled trial. J Sports Sci. 2018; 36(14):1663-1672. PubMed ID: 29192837 doi:10.1080/02640414. 2017.1409609

63. Lovell R, Knox M, Weston M, Siegler JC, Brennan S, Marshall PWM. Hamstring injury prevention in soccer: before or after training? Scand J Med Sci Sport. 2018;28(2):658-666. doi:10.1111/sms. 12925

64. Mendiguchia J, Conceição F, Edouard P, et al. Sprint versus isolated eccentric training: comparative effects on hamstring architecture and performance in soccer players. PLoS One. 2020; 15(2):e0228283. PubMed ID: 32045411 doi:10.1371/journal.pone. 0228283

65. Mjølsnes R, Arnason A, Østhagen T, Raastad T, Bahr R. A 10-week randomized trial comparing eccentric vs concentric hamstring strength training in well-trained soccer players. Scand J Med Sci Sport. 2004;14(5):311-317. doi:10.1046/j.1600-0838.2003.00367.x

66. Ribeiro-Alvares J, Marques VB, Vaz MA, Baroni BM. Four weeks of Nordic hamstring exercise reduce muscle injury risk factors in young adults. J Strength Cond Res. 2018;32(5):1254-1262. PubMed ID: 28459795 doi:10.1519/JSC.0000000000001975

67. Suarez-Arrones L, Lara-Lopez P, Rodriguez-Sanchez P, et al. Dissociation between changes in sprinting performance and Nordic hamstring strength in professional male football players. PLoS One. 2019;14(3):e0213375. doi:10.1371/journal.pone.0213375

68. Small K, Mcnaughton L, Greig M, Lovell R. Effect of timing of eccentric hamstring strengthening exercises during soccer training: implications for muscle fatigability. J Strength Cond Res. 2009;23(4): 1077-1083. PubMed ID: 19528859 doi:10.1519/JSC.0b013e3181 94df5c

69. Schache AG, Dorn TW, Blanch PD, Brown NAT, Pandy MG. Mechanics of the human hamstring muscles during sprinting. Med Sci Sports Exerc. 2012;44(4):647-658. PubMed ID: 21912301 doi: 10.1249/MSS.0b013e318236a3d2

70. Guex K, Millet GP. Conceptual framework for strengthening exercises to prevent hamstring strains. Sport Med. 2013;43(12): 1207-1215. doi:10.1007/s40279-013-0097-y

71. Van Dyk N, Bahr R, Whiteley R, et al. Hamstring and quadriceps isokinetic strength deficits are weak risk factors for hamstring strain injuries. Am J Sports Med. 2016;44(7):1789-1795. doi:10.1177/ 0363546516632526

72. Reilly T, Morris T, Whyte G. The specificity of training prescription and physiological assessment: a review. J Sports Sci. 2009; 27(6):575-589. PubMed ID: 19340630 doi:10.1080/026404109027 29741

73. Lovell R, Whalan M, Marshall PWM, Sampson JA, Siegler JC, Buchheit M. Scheduling of eccentric lower limb injury prevention exercises during the soccer micro-cycle: which day of the week? Scand J Med Sci Sport. 2018;28(10):2216-2225. doi:10.1111/sms. 13226

74. Severo-Silveira L, Dornelles MP, Lima-E-Silva FX, et al. Progressive workload periodization maximizes effects of Nordic hamstring exercise on muscle injury risk factors. J strength Cond Res. 2018;28(7): 1775-1783. doi:10.1519/JSC.0000000000002849

75. Presland JD, Timmins RG, Bourne MN, Williams MD, Opar DA. The effect of Nordic hamstring exercise training volume on biceps femoris long head architectural adaptation. Scand J Med Sci Sport. 2018; 28(7):1775-1783. doi:10.1111/sms. 13085

76. Blazevich AJ, Gill ND, Zhou S. Intra- and intermuscular variation in human quadriceps femoris architecture assessed in vivo. J Anat. 2006; 
209(3):289-310. PubMed ID: 16928199 doi:10.1111/j.1469-7580. 2006.00619.x

77. Timmins RG, Shield AJ, Williams MD, Lorenzen C, Opar DA. Biceps femoris long head architecture: a reliability and retrospective injury study. Med Sci Sports Exerc. 2015;47(5):905-913. PubMed ID: 25207929 doi:10.1249/MSS.0000000000000507

78. Lieber RL, Bodine-Fowler SC. Skeletal muscle mechanics: implications for rehabilitation. Phys Ther. 1993;73(12):844-856. PubMed ID: 8248293 doi:10.1093/ptj/73.12.844

79. Lieber RL, Fridén J. Muscle damage is not a function of muscle force but active muscle strain. J Appl Physiol. 1993;74(2):520-526. PubMed ID: 8458765 doi:10.1152/jappl.1993.74.2.520

80. Morgan DL. New insights into the behavior of muscle during active lengthening. Biophys J. 1990;57(2):209-221. PubMed ID: 2317547 doi:10.1016/S0006-3495(90)82524-8
81. Geremia JM, Baroni BM, Lanferdini FJ, Bini RR, Sonda FC, Vaz MA. Time course of neuromechanical and morphological adaptations to triceps surae isokinetic eccentric training. Phys Ther Sport. 2018;34:84-91. PubMed ID: 30248491 doi:10.1016/j.ptsp.2018. 09.003

82. Al Attar WSA, Soomro N, Sinclair PJ, Pappas E, Sanders RH. Effect of injury prevention programs that include the Nordic hamstring exercise on hamstring injury rates in soccer players: a systematic review and meta-analysis. Sport Med. 2017;47(5):907-916. doi:10. 1007/s40279-016-0638-2

83. Goode AP, Reiman MP, Harris L, et al. Eccentric training for prevention of hamstring injuries may depend on intervention compliance: a systematic review and meta-analysis. $\mathrm{Br}$ J Sports Med. 2015;49(6):349-356. PubMed ID: 25227125 doi:10.1136/bjsports2014-093466 